\title{
Antitumor activity of bee pollen polysaccharides from Rosa rugosa
}

\author{
BO WANG ${ }^{1,2}$, QIYU DIAO ${ }^{3}$, ZHONGYU ZHANG $^{1}$, YANG LIU ${ }^{1}$, QIPIN GAO ${ }^{1}$, YIFA ZHOU ${ }^{1}$ and SHANSHAN LI $^{1}$ \\ ${ }^{1}$ School of Life Sciences, Northeast Normal University, Jilin 130024; ${ }^{2}$ College of Chemistry and Biology, \\ Beihua University, Jilin $132013 ;{ }^{3}$ Feed Research Institute, Chinese Academy \\ of Agricultural Sciences, Beijing 100081, P.R. China
}

Received November 13, 2012; Accepted January 29, 2013

DOI: $10.3892 / \mathrm{mmr} .2013 .1382$

\begin{abstract}
In the present study, bee pollen polysaccharides from Rosa rugosa (WRPP) were extracted and fractionated. WRPP were purified to neutral (WRPP-N) and acidic polysaccharides (WRPP-1, WRPP-2) with DEAE-Cellulose. WRPP-N were mainly composed of glucose, mannose, arabinose and galactose, indicating the existence of glucan, arabinogalactan (AG) and mannoglucan. WRPP-1 mainly consisted of rhamnose $(3.0 \%)$, galacturonic acid $(12.4 \%)$, galactose $(24.7 \%)$ and arabinose (53.9\%), and contained a large proportion of AGs. WRPP-2 consisted of rhamnose (7.8\%), galacturonic acid $(23.0 \%)$, galactose $(15 \%)$ and arabinose $(48.7 \%)$, while WRPP-2 contained more galacturonic acid compared to WRPP-1. WRPP-1 and WRPP-2 were composed by type I rhamnogalacturonan (RG-I), homogalacturonan (HG) and AG fragments, while WRPP-2 contained more HG and RG-I. All the fractions had significant anti-proliferative activity in HT-29 and HCT116 cells; the neutral and acidic fractions were shown to have significant synergistic effects which accounted for the antitumor activity of bee pollen polysaccharides from Rosa rugosa in vitro.
\end{abstract}

\section{Introduction}

Bee pollen is the male gametophyte of gymnosperms and angiosperms, which contains all the necessary nutrients for plant growth and development. Bee pollen is known to be a natural and healthy food that improves human immunity (1). Bee pollen polysaccharides exhibit antitumor (2) and immunomodulating activities $(3,4)$. Previous studies have demonstrated that pollen significantly inhibits tumor growth and enhances immunomodulating activity, while alleviating the distress of chemotherapy in cancer patients $(5,6)$.

Correspondence to: Dr Shanshan Li, School of Life Sciences, Northeast Normal University, 5268 Renmin Street, Changchun, Jilin 130024, P.R. China

E-mail: liss576@nenu.edu.cn

Key words: polysaccharide, bee pollen, Rosa rugosa, fractionation, antitumor
Polysaccharides constitute one group of the main components of plants, and have complex structural features including type I (RG-I) and type II rhamnogalacturonan (RG-II), homogalacturonan (HG) and arabinogalactan (AG). Polysaccharides have been reported to possess a broad spectrum of biological activities, particularly antitumor (7-10) and immunomodulating activities (11-16). Rosa rugosa is a common type of plants in China, and its bee pollen has been used as an immunological food for a long time. Polysaccharides are the main active components in bee pollen. However, there is limited information regarding the fractionation and antitumor activity of bee pollen polysaccharides. To the best of our knowledge, this is the first study to investigate the total fractionation and antitumor activity of bee pollen polysaccharides from Rosa rugosa.

\section{Materials and methods}

Materials. Bee pollen from Rosa rugosa was kindly provided by the Feed Research Institute Chinese Academy of Agricultural Sciences (Beijing, China). Sepharose ${ }^{\circledR}$ CL-6B was purchased from Sigma-Aldrich (St. Louis, MO, USA). Dulbecco's modified Eagle's medium:Nutrient Mixture F12 (DMEM/F12) medium and calf serum were purchased from Gibco (Carlsbad, CA, USA). Trypsin was obtained from Amersco (Framingham, MA, USA), 3-(4, 5-dimethylthiazol2-yl)-2,5-diphenyl tetrazolium bromide (MTT) from Sigma (St. Louis, MO, USA), and penicillin/streptomycin from the Tianjin Hao Yang Biological Manufacture Co., Ltd. (Tianjin, China). All the additional analytical grade chemicals and reagents were produced in China.

Analytical methods. Total carbohydrate contents were determined using the phenol-sulphuric acid method (16,17). Uronic acid contents were determined using the m-hydroxydiphenyl method $(16,18)$. All the gel permeation and anion exchange chromatographies were monitored by assaying total sugar and uronic acid contents. Sugar composition analysis was performed as described by Zhang et al (15) and Yu et al (19). Each polysaccharide sample (2 $\mathrm{mg}$ ) was hydrolyzed with $2 \mathrm{M}$ trifluoroacetic acid (TFA) at $120^{\circ} \mathrm{C}$ for $2 \mathrm{~h}$. The monosaccharide derivatives were released using 1-phenyl-3-methyl-5-pyrazolone and analyzed on a DIKMA Inertsil ODS-3 column $(4.6 \times 150 \mathrm{~mm})$ connected to a Shimadzu high performance liquid chromatography (HPLC) system (LC-10ATvp pump and SPD-10AVD UV-VIS detector). 

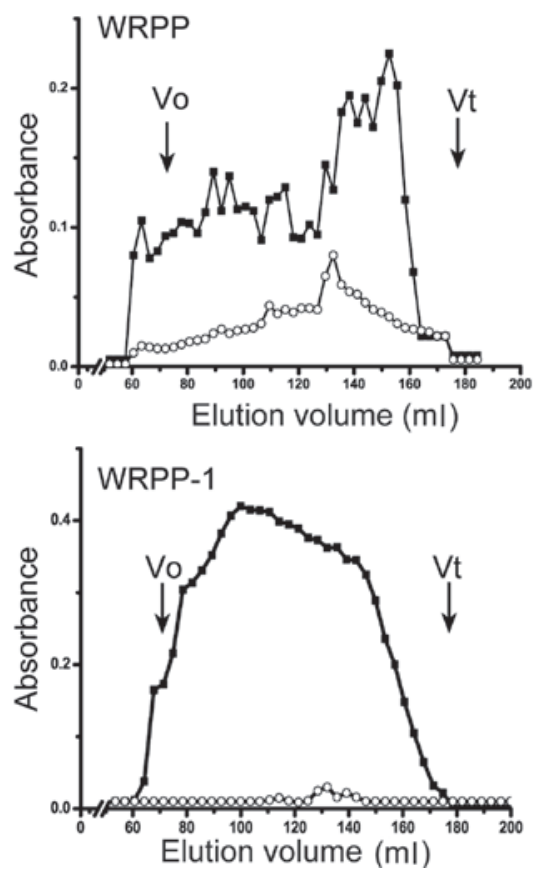
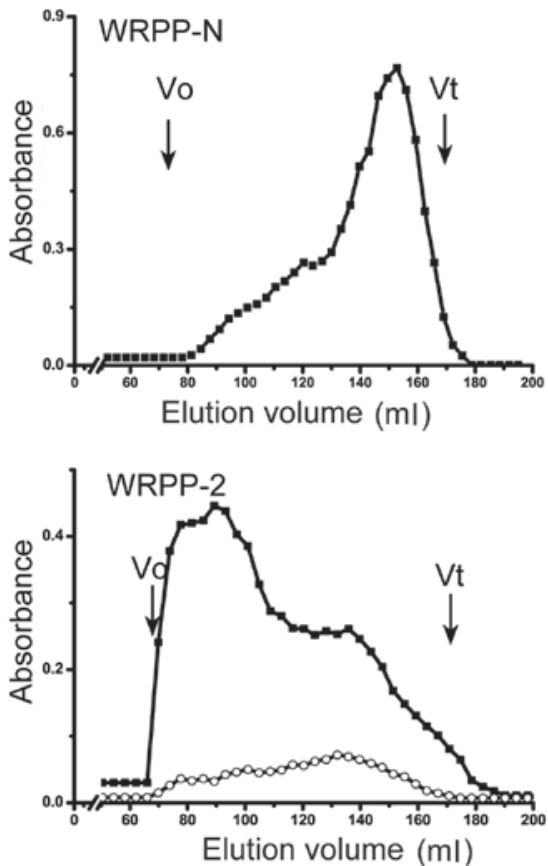

Figure 1. Molecular weight distributions of polysaccharide fractions using Sepharose CL-6B. $\mathbf{m}$, total sugars; O, uronic acid. Vo, out volume; Vt, total volume.

Cell culture. The cell lines HT-29 and HCT116 were obtained from the American Type Culture Collection (ATCC; Manassas, VA, USA). HT-29 cells were cultured in DMEM/F12 medium supplemented with $10 \%$ calf serum and $100 \mathrm{IU} / \mathrm{ml}$ penicillin and streptomycin. HCT116 cells were cultured in Iscove's modified Dulbecco's medium (IMDM) supplemented with $10 \%$ calf serum and $100 \mathrm{IU} / \mathrm{ml}$ penicillin and streptomycin. The cells were incubated at $37^{\circ} \mathrm{C}$ in a humidified atmosphere of $5 \% \mathrm{CO}_{2}$ in air.

Cell proliferation assay. HT-29 and HCT116 cells were seeded in a 96-well plate $\left(\right.$ Costar $\left.^{\circledR}\right)$ at a density of $1 \times 10^{4}$ cells/well, adhered for $24 \mathrm{~h}$, and then treated for $72 \mathrm{~h}$ with the polysaccharide fractions at varying concentrations (7). Control cells were similarly treated without the polysaccharides. The media were removed and $100 \mu \mathrm{l} /$ well of MTT solution $(0.5 \mathrm{mg} / \mathrm{ml})$ were added. The supernatants were discarded after a 4-h incubation. The resulting formazan was dissolved in $100 \mu 1 \mathrm{DMSO}$ and the absorbance at $570 \mathrm{~nm}$ was measured using a microplate reader (Bio-Rad, Hercules, CA, USA). All the experiments were performed in triplicate and repeated at least three times. Cell proliferation under all the conditions was expressed as a percentage of the control, which was set at $100 \%$.

Statistical analysis. Data were presented as the means \pm standard deviation (SD). Statistical analysis was performed using SPSS version 17.0 software. One-way analysis of variance (ANOVA) test was used to perform a statistical comparison between the treatment and control groups. $\mathrm{P}<0.05$ and $<0.01$ were considered to indicate a statistically significant difference.

\section{Results}

Isolation and fractionation of bee pollen polysaccharides. The water-soluble polysaccharides were extracted from the bee pollen of Rosa rugosa with hot water. Following precipitation by the addition of 4 volumes of $95 \%$ ethanol, a total polysaccharides fraction, referred to as WRPP (water-soluble Rosa rugosa bee pollen polysaccharides), was obtained with a yield of $4.6 \%(\mathrm{w} / \mathrm{w})$. WRPP contained $58.1 \%$ total sugar and $13.3 \%$ uronic acid. WRPP was a water-soluble light yellow powder. Sugar composition analysis by HPLC indicated that WRPP consisted of galactose (Gal) (21.4\%), arabinose (Ara) $(47.9 \%)$, rhamnose (Rha) $(3.4 \%)$, galacturonic acid (GalA) $(12.1 \%)$, glucose (Glc) $(11.6 \%)$, mannose (Man) $(2.6 \%)$ and glucoronic acid (GlcA) $(1.0 \%)$.

WRPP contain neutral and acidic polysaccharides and showed a wide molecular weight distribution using Sepharose CL-6B (Fig. 1). To fractionate WRPP, it was applied on anionexchange chromatography (Fig. 2). The elution profiles on DEAE-Cellulose column are shown in Fig. 3. Three fractions of WRPP-N, WRPP-1 and WRPP-2 were eluted with distilled water, 0.2 and $0.4 \mathrm{M} \mathrm{NaCl}$, respectively. The molecular distribution on Sepharose CL-6B was analyzed (Fig. 1). WRPP-N exhibited one main peak near the total volume, while small curves prior to the main peak also existed, suggesting a mixture of polysaccharides with different molecular weights. WRPP-1 showed wide distribution from the out volume to the total volume of Sepharose CL-6B, while uronic acid showed a small peak. WRPP-2 exhibited a main peak near the out volume, while an additional peak of rich uronic acid appeared near the total volume.

Composition analysis of the polysaccharide fractions. The monosaccharide compositions and yields of the collected fractions are listed in Table I. WRPP-N was composed of Glc (34.3\%), Gal (25.0\%), Ara (36.7\%) and Man (4.0\%), while uronic acid was not detected. The iodine test suggested that no starch was present, while WRPP-N was composed of glucan, arabinogalactans (AGs) and few mannoglucans. 
Table I. Yields and structral features of the collected fractions.

\begin{tabular}{|c|c|c|c|c|c|c|c|c|}
\hline \multirow[b]{2}{*}{ Fraction } & \multirow[b]{2}{*}{ Yield (\%) } & \multicolumn{7}{|c|}{ Monosaccharide composition (\%) } \\
\hline & & Rha & GalA & Gal & Ara & Man & Glc & GlcA \\
\hline WRPP & $4.6^{\mathrm{a}}$ & 3.4 & 12.1 & 21.4 & 47.9 & 2.6 & 11.6 & 1.0 \\
\hline WRPP-N & $26.9^{\mathrm{b}}$ & 0 & 0 & 25.0 & 36.7 & 4.0 & 34.3 & 0 \\
\hline WRPP-1 & $21.4^{\mathrm{b}}$ & 3.0 & 12.4 & 24.7 & 53.9 & 1.9 & 3.0 & 1.1 \\
\hline WRPP-2 & $13.1^{\mathrm{b}}$ & 7.8 & 23.0 & 15.0 & 48.7 & 1.7 & 2.8 & 0.9 \\
\hline
\end{tabular}

${ }^{a}$ Yield as $\%$ of bee pollen dry weight; byield as $\%$ of fraction applied to column. Rha, rhamnose; GalA, galacturonic acid; Gal, galactose; Ara, arabinose; Man, mannose; Glc, glucose; GlcA, glucoronic acid.

\section{Bee pollen from Rosa rugosa}

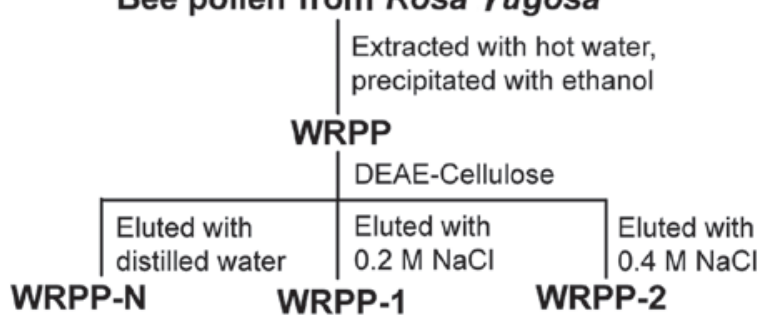

Figure 2. Fractionation scheme of bee pollen polysaccharides from Rosa rugosa.

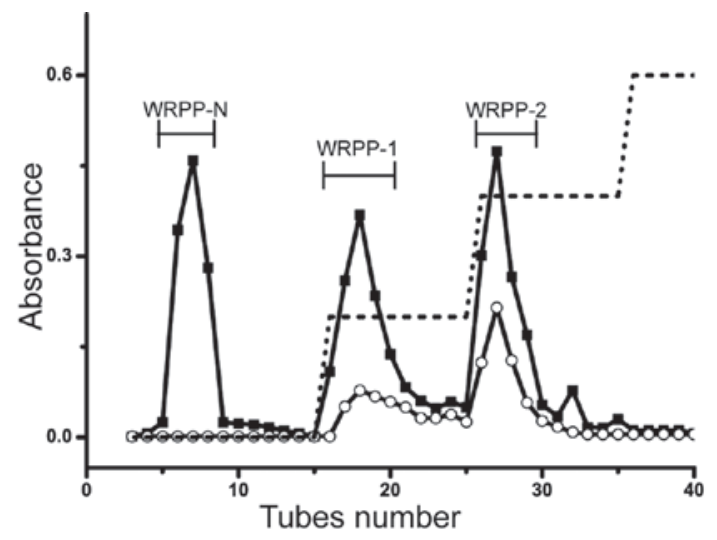

Figure 3. Elution profiles of WRPP on DEAE-Cellulose by a stepwise gradient of $\mathrm{NaCl}$. total sugars; $\mathrm{O}$, uronic acid; dashed line, concentration of $\mathrm{NaCl}$.

WRPP-1 consisted of Rha (3.0\%), GalA (12.4\%), Gal (24.7\%) and Ara (53.9\%), with minor amounts of Man, Glc and GlcA being detected (Table I). The amounts of Gal and Ara were $>70 \%$ of total sugars. The ratio of Rha/GalA was 0.24 , which was within the RG-I range of 0.05-1.0, defined by Schols and Voragen (20) and Yu et al (19). These results indicated small amounts of RG-I and HG domains, while a large proportion of $\mathrm{AG}$ and arabinan fragments was evident.

WRPP-2 consisted of Rha (7.8\%), GalA (23.0\%), Gal (15.0\%) and Ara (48.7\%), with minor amounts of Glc, GlcA and Man being detected. The ratio of Rha/GalA was $\sim 0.34$, suggesting the presence of RG-I, HG and AG.

WRPP-1 and WRPP-2 were mainly composed of Gal, Ara, Rha and GalA. Both had a large proportion of Gal and Ara,
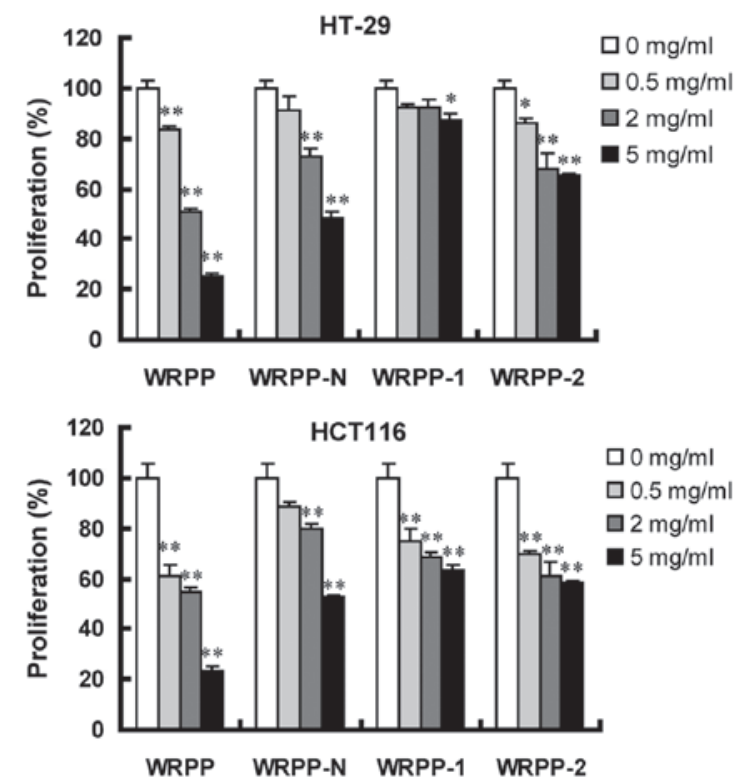

Figure 4. Anti-proliferative effects of bee pollen polysaccharides from Rosa rugosa on HT-29 and HCT116 cells. HT-29 and HCT116 cells were treated with various polysaccharides at varying concentrations for $72 \mathrm{~h}$, as indicated. Cell proliferation was determined using MTT assay. Columns, means from three independent experiments; bars, $\mathrm{SD} .{ }^{*} \mathrm{P}<0.05,{ }^{* *} \mathrm{P}<0.01$.

suggesting the existence of AG fragments. The amounts of GalA in WRPP-1 and WRPP-2 were 12.4 and 23\%, respectively; one explanatory reason could be the elution order from the DEAE-Cellulose column. WRPP-2 contained more RG-I and HG domains compared to WRPP-1, while WRPP-1 contained more AG fragments compared to WRPP-2.

Antitumor effects in vitro. Human colon cancer HT-29 and HCT116 cells were treated with varying concentrations of various bee pollen polysaccharides from Rosa rugosa for $72 \mathrm{~h}$. Cell proliferation was assessed using MTT assay. Following a 72 -h treatment with total polysaccharides WRPP, cell proliferation was significantly inhibited in a dose-dependent manner. All the sub-fractions of WRPP also showed a concentration-dependent proliferation-inhibitory effect on HT-29 and HCT116 cells (Fig. 4). Notably, all the sub-fractions were less effective compared to the total fraction WRPP, suggesting that sub-fractions have a synergistic effect, which accounted for the anti-proliferative activity of 
bee pollen polysaccharides from Rosa rugosa on HT-29 and HCT116 cells in vitro.

\section{Discussion}

In the present study, bee pollen polysaccharides from Rosa rugosa were extracted and fractionated. Total WRPP were purified to neutral (WRPP-N) and acidic polysaccharides (WRPP-1, WRPP-2). Acidic fractions contained different amounts of RG-I, HG and AG fragments. All the fractions inhibited the proliferation of HT-29 and HCT116 cells in a dose-dependent manner in vitro, indicating a potential antitumor activity. Sub-fractions showed a significant synergistic effect. To the best of our knowledge, this is the first study investigating the fractionation and antitumor activity of bee pollen polysaccharides from Rosa rugosa.

\section{Acknowledgements}

This study was supported by the Fundamental Research Funds for the Central Universities (no. 09SSXT125).

\section{References}

1. Kroyer G and Hegedus N: Evaluation of bioactive properties of pollen extracts as functional dietary food supplement. Innov Food Sci Emerg Technol 2: 171-174, 2001.

2. Yang X, Guo D, Zhang J and Wu M: Characterization and antitumor activity of pollen polysaccharide. Int Immunopharmacol 7: 427-434, 2007.

3. Li F, Yuan Q and Rashid F: Isolation, purification and immunobiological activity of a new water-soluble bee pollen polysaccharide from Crataegus pinnatifida Bge. Carbohydr Polym 78: 80-88, 2009.

4. Brecker L, Wicklein D, Moll H, Fuchs EC, Becker WM and Petersen A: Structural and immunological properties of arabinogalactan polysaccharides from pollen of timothy grass (Phleum pratense L.). Carbohydr Res 340: 657-663, 2005.

5. Gao Y, Hu F, Zhu W and Li Y: Study on the antitumor activity of propolis and bee pollen and royal jelly. J Bee 7: 3-4, 2003.
6. Wang W, Hu J and Shen W: Antitumor activity and mechanism of pollen. Chin Beekeep 36: 1-3, 1986.

7. Cheng H, Li S, Fan Y, et al: Comparative studies of the antiproliferative effects of ginseng polysaccharides on HT-29 human colon cancer cells. Med Oncol 28: 175-181, 2011.

8. Fan Y, Cheng H, Liu D, et al: The inhibitory effect of ginseng pectin on L-929 cell migration. Arch Pharm Res 33: 681-689, 2010.

9. Xu C, Liu Y, Yuan G and Guan M: The contribution of side chains to antitumor activity of a polysaccharide from Codonopsis pilosula. Int J Biol Macromol 50: 891-894, 2012.

10. Zhao H, Li Y, Wang Y, et al: Antitumor and immunostimulatory activity of a polysaccharide-protein complex from Scolopendra subspinipes mutilans L. Koch in tumor-bearing mice. Food Chem Toxicol 50: 2648-2655, 2012.

11. Inngjerdingen KT, Kiyohara H, Matsumoto T, et al: An immunomodulating pectic polymer from Glinus oppositifolius. Phytochemistry 68: 1046-1058, 2007.

12. Inngjerdingen M, Inngjerdingen KT, Patel TR, et al: Pectic polysaccharides from Biophytum petersianum Klotzsch, and their activation of macrophages and dendritic cells. Glycobiology 18: 1074-1084, 2008.

13. Nergard CS, Matsumoto T, Inngjerdingen M, et al: Structural and immunological studies of a pectin and a pectic arabinogalactan from Vernonia kotschyana Sch. Bip. ex Walp. (Asteraceae). Carbohydr Res 340: 115-130, 2005.

14. Ni W, Zhang X, Bi H, et al: Preparation of a glucan from the roots of Rubus crataegifolius Bge. and its immunological activity. Carbohydr Res 344: 2512-2518, 2009.

15. Zhang X, Li S, Sun L, et al: Further analysis of the structure and immunological activity of an RG-I type pectin from Panax ginseng. Carbohydr Polym 89: 519-525, 2012.

16. Zhang X, Yu L, Bi H, et al: Total fractionation and characterization of the water-soluble polysaccharides isolated from Panax ginseng C. A. Meyer. Carbohydr Polym 77: 544-552, 2009.

17. Dubois M, Gilles KA, Hamilton JK, Rebers PA and Smith F: Colorimetric method for determination of sugars and related substances. Anal Chem 28: 350-356, 1956.

18. Blumenkrantz N and Asboe-Hansen G: New method for quantitative determination of uronic acids. Anal Biochem 54: 484-489, 1973.

19. Yu L, Zhang X, Li S, et al: Rhamnogalacturonan I domains from ginseng pectin. Carbohydr Polym 79: 811-817, 2010.

20. Schols HA and Voragen A: Complex pectins: structure elucidation using enzymes. Progress Biotechnol 14: 3-19, 1996. 